Acta Crystallographica Section E

Structure Reports

Online

ISSN 1600-5368

\section{Redetermined structure of diphenyl- phosphonimidotriphenylphosphorane: location of the hydrogen atoms and analysis of the intermolecular interactions}

\section{Richard Betz, ${ }^{*}$ Thomas Gerber, Eric Hosten and Henk Schalekamp}

Nelson Mandela Metropolitan University, Summerstrand Campus, Department of Chemistry, University Way, Summerstrand, PO Box 77000, Port Elizabeth, 6031, South Africa

Correspondence e-mail: richard.betz@webmail.co.za

Received 25 March 2011; accepted 28 March 2011

Key indicators: single-crystal X-ray study; $T=200 \mathrm{~K}$; mean $\sigma(\mathrm{C}-\mathrm{C})=0.003 \AA$; $R$ factor $=0.027 ; w R$ factor $=0.076$; data-to-parameter ratio $=14.7$.

The title compound, $\mathrm{C}_{30} \mathrm{H}_{25} \mathrm{NOP}_{2}$, is a bulky phosphazene derivative. Its previous crystal structure [Cameron et al. (1979). Acta Cryst. B35, 1373-1377] is confirmed and its H atoms have been located in the present study. The formal $\mathrm{P}=\mathrm{N}$ double bond is about $0.05 \AA$ shorter than the $\mathrm{P}-\mathrm{N}$ single bond and the large $\mathrm{P}=\mathrm{N}-\mathrm{P}$ bond angle reflects the steric strain in the molecule. An intramolecular $\mathrm{C}-\mathrm{H} \cdots \mathrm{O}$ interaction occurs. In the crystal, short $\mathrm{C}-\mathrm{H} \cdots \mathrm{O}$ contacts connect the molecules into chains propagating in [011], which are cross-linked via $\mathrm{C}-\mathrm{H} \cdots \pi$ interactions, generating a threedimensional network. Aromatic $\pi-\pi$ stacking also occurs [shortest centroid-centroid separation $=3.6012(11) \AA$ ]

\section{Related literature}

For the previous structure determination, see: Cameron et al. (1979). For graph-set analysis of hydrogen bonds, see: Etter et al. (1990); Bernstein et al. (1995).

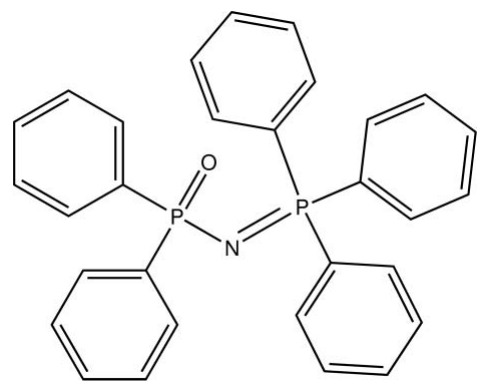

\section{Experimental}

Crystal data

$\mathrm{C}_{30} \mathrm{H}_{25} \mathrm{NOP}_{2}$

$M_{r}=477.45$

Orthorhombic, Pna2 1

$a=17.6607$ (12) ̊

$b=15.1593(10) \AA$

$c=8.9192(6) \AA$

$V=2387.9(3) \AA^{3}$

$Z=4$

Mo $K \alpha$ radiation

$\mu=0.21 \mathrm{~mm}^{-1}$

$T=200 \mathrm{~K}$

$0.88 \times 0.42 \times 0.31 \mathrm{~mm}$

Data collection

Bruker APEXII CCD

diffractometer

12511 measured reflections

4498 independent reflections 4348 reflections with $I>2 \sigma(I)$ $R_{\text {int }}=0.021$

Refinement

$R\left[F^{2}>2 \sigma\left(F^{2}\right)\right]=0.027$

$w R\left(F^{2}\right)=0.076$

$S=1.11$

4498 reflections

307 parameters

1 restraint

$\mathrm{H}$-atom parameters constrained

$\Delta \rho_{\max }=0.21{\mathrm{e} \AA^{-3}}^{-3}$

$\Delta \rho_{\text {min }}=-0.25{\mathrm{e} \AA^{-3}}^{-3}$

Absolute structure: Flack (1983), 1332 Friedel pairs

Flack parameter: -0.03 (6)

Table 1

Selected geometric parameters $\left(\AA,^{\circ}\right)$.

\begin{tabular}{llll}
\hline $\mathrm{P} 1-\mathrm{N} 1$ & $1.6014(13)$ & $\mathrm{P} 2-\mathrm{N} 1$ & $1.5532(13)$ \\
$\mathrm{P} 2-\mathrm{N} 1-\mathrm{P} 1$ & $146.35(12)$ & & \\
\hline
\end{tabular}

Table 2

Hydrogen-bond geometry $\left(\AA{ }^{\circ}\right)$.

\begin{tabular}{lllll}
\hline$D-\mathrm{H} \cdots A$ & $D-\mathrm{H}$ & $\mathrm{H} \cdots A$ & $D \cdots A$ & $D-\mathrm{H} \cdots A$ \\
\hline $\mathrm{C} 32-\mathrm{H} 32 \cdots \mathrm{O} 1$ & 0.95 & 2.34 & $3.257(2)$ & 163 \\
$\mathrm{C} 43-\mathrm{H} 43 \cdots \mathrm{O} 1^{\mathrm{i}}$ & 0.95 & 2.34 & $3.257(2)$ & 162 \\
$\mathrm{C} 45-\mathrm{H} 45 \cdots C g 1^{\mathrm{ii}}$ & 0.95 & 2.92 & $3.846(2)$ & 165 \\
$\mathrm{C} 55-\mathrm{H} 55 \cdots C g 2^{\text {iii }}$ & 0.95 & 2.73 & $3.644(2)$ & 163 \\
\hline
\end{tabular}

Symmetry codes: (i) $-x+\frac{1}{2}, y-\frac{1}{2}, z+\frac{1}{2}$; (ii) $x, y, z+1$; (iii) $-x,-y, z-\frac{1}{2}$.

Data collection: APEX2 (Bruker, 2010); cell refinement: SAINT (Bruker, 2010); data reduction: $S A I N T$; program(s) used to solve structure: SHELXS97 (Sheldrick, 2008); program(s) used to refine structure: SHELXL97 (Sheldrick, 2008); molecular graphics: ORTEPIII (Farrugia, 1997) and Mercury (Macrae et al., 2006); software used to prepare material for publication: SHELXL97 and PLATON (Spek, 2003).

The authors thank Mr Gerhard Schneeberger for helpful discussions.

Supplementary data and figures for this paper are available from the IUCr electronic archives (Reference: HB5826). 


\section{organic compounds}

\section{References}

Bernstein, J., Davis, R. E., Shimoni, L. \& Chang, N.-L. (1995). Angew. Chem. Int. Ed. Engl. 34, 1555-1573.

Bruker (2010). APEX2 and SAINT Bruker AXS Inc., Madison, USA.

Cameron, A. F., Cameron, I. R. \& Keat, R. (1979). Acta Cryst. B35, 1373-1377.

Etter, M. C., MacDonald, J. C. \& Bernstein, J. (1990). Acta Cryst. B46, 256-262.
Farrugia, L. J. (1997). J. Appl. Cryst. 30, 565.

Flack, H. D. (1983). Acta Cryst. A39, 876-881.

Macrae, C. F., Edgington, P. R., McCabe, P., Pidcock, E., Shields, G. P., Taylor, R., Towler, M. \& van de Streek, J. (2006). J. Appl. Cryst. 39, 453-457. Sheldrick, G. M. (2008). Acta Cryst. A64, 112-122.

Spek, A. L. (2003). J. Appl. Cryst. 36, 7-13. 


\section{supporting information}

Acta Cryst. (2011). E67, o1028-o1029［doi:10.1107/S1600536811011500]

\section{Redetermined structure of diphenylphosphonimidotriphenylphosphorane: location of the hydrogen atoms and analysis of the intermolecular interactions}

\section{Richard Betz, Thomas Gerber, Eric Hosten and Henk Schalekamp}

\section{S1. Comment}

For many main group elements as well as transition and rare earth metals, preferred coordination numbers in coordination compounds are apparent. While coordination numbers of 4, 6 and 8 have been found to be dominant in most cases and, as a consequence, vast structural information has been collected for such compounds in solution and in the solid state, information about other coordination numbers is comparatively limited. Especially for smaller coordination numbers the literature is scant or hitherto completely unknown for many elements. One reason for this certainly is that sometimes challenging synthesis procedures have to be followed and, thus, a general but simple synthetic protocol is desireable. Since such compounds may act as versatile and potent catalysts in many industrial processes and might even show interesting pharmacological properties, we were interested in developing an easy-access-route for their synthesis. Applying bulky ligands might open up a pathway in this aspect. In order to be able to compare metrical parameters in envisioned reaction products, we determined the crystal structure of the title compound. The latter one has already been reported earlier (Cameron et al. (1979)), however, no hydrogen atoms were included in the refinement thus ruling out the possibility to assess the role of $\mathrm{C}-\mathrm{H} \cdots X$ contacts.

The length of the N-P bonds deviate by $0.05 \AA$ with the - formal - P-N-double bond found at around $1.55 \AA$. The $\mathrm{P}-\mathrm{N}-$ $\mathrm{P}$ angle was measured at more than $146^{\circ}$. The marked widening of this angle in comparison to the value expected for a $s p^{2}$-hybridized nitrogen atom can be explained by the repulsive interaction of the phenyl-moieties on both $\mathrm{P}$ atoms. The phenyl groups on each phosphorus atom are approximately orientated perpendicular to each other. The least-squares planes defined by their carbon atoms intersect at an angle of 82.19 (6) ${ }^{\circ}$ in case of the $\mathrm{P}(\mathrm{O}) \mathrm{Ph}_{2}$-moiety and at angles of $79.82(5)^{\circ}, 80.91(6)^{\circ}$ and $83.28(6)^{\circ}$, respectively, in case of the $\mathrm{PPh}_{3}$-moiety. Due to the formation of an intramolecular $\mathrm{C}-\mathrm{H} \cdots \mathrm{O}$ contact (see below), the least-squares plane defined by the $\mathrm{P}(\mathrm{O})-\mathrm{N}-\mathrm{P}$ motif encloses an angle of only 29.40 (9) ${ }^{\circ}$ with one of the aromatic carbocycles on the $\mathrm{PPh}_{3}$-moiety (Fig. 1). For the same reason, both phenyl groups of the $\mathrm{P}(\mathrm{O}) \mathrm{Ph}_{2}$-moiety adopt a slightly ecliptic conformation with respect to the $\mathrm{P}(\mathrm{O})$ motif, the respective dihedral angles were found at about $19^{\circ}$ and $26^{\circ}$.

In the crystal structure, intermolecular $\mathrm{C}-\mathrm{H} \cdots \mathrm{O}$ contacts are present whose range falls by more than $0.3 \AA$ below the sum of van-der-Waals radii of the atoms participating. These can be observed between one of the $\mathrm{H}$ atoms in metaposition of a phenyl group on the $\mathrm{PPh}_{3}$-moiety and the $\mathrm{O}$ atom of the $\mathrm{P}(\mathrm{O}) \mathrm{Ph}_{2}$-moiety and connect the molecules to infinte chains along $\left[\begin{array}{lll}0 & 1 & 1\end{array}\right]$ (Fig. 2). Furthermore, intramolecular $\mathrm{C}-\mathrm{H} \cdots \mathrm{O}$ contacts invariably involving hydrogen atoms in ortho-position on one of the phenyl groups of the $\mathrm{PPh}_{3}$-moiety as well as both phenyl groups of the $\mathrm{P}(\mathrm{O}) \mathrm{Ph}_{2}$-moiety are present. However, the latter two ones are not very pronounced. Additionally, a set of $\mathrm{C}-\mathrm{H} \cdots \pi$ contacts are evident involving $\mathrm{H}$ atoms and aromatic systems both on the $\mathrm{PPh}_{3}$-moiety as well as the $\mathrm{P}(\mathrm{O}) \mathrm{Ph}_{2}$-moiety. Their details are listed in Table 1 (with $C_{\mathrm{g}}(1)=\mathrm{C} 41 \cdots \mathrm{C} 46, C_{\mathrm{g}}(2)=\mathrm{C} 31 \cdots \mathrm{C} 36$ and $\left.C_{\mathrm{g}}(3)=\mathrm{C} 11 \cdots \mathrm{C} 16\right)$. In total, the $\mathrm{C}-\mathrm{H} \cdots \mathrm{O}$ contacts as well as 
the $\mathrm{C}-\mathrm{H} \cdots \pi$ contacts connect the molecules to a three dimensional network. In terms of graph-set analysis (Etter et al. (1990); Bernstein et al. (1995)), the intermolecular $\mathrm{C}-\mathrm{H} \cdots \mathrm{O}$ contacts can be assigned a $C^{1}{ }_{1}(8)$ descriptor on the unitary level while the intramolecular $\mathrm{C}-\mathrm{H} \cdots \mathrm{O}$ contact involving the phenyl group of the $\mathrm{PPh}_{3}$-moiety necessitates a $S_{1}{ }_{1}(7)$ descriptor. For the other two intramolecular $\mathrm{C}-\mathrm{H} \cdots \mathrm{O}$ contacts, a $S^{1}{ }_{1}(5)$ each is feasible. An analysis of $C_{\mathrm{g}} \cdots C_{\mathrm{g}}$ interactions shows the closest distance between two centers of gravity to occur between a phenyl group on the $\mathrm{PPh}_{3}$-moiety and a phenyl group on the $\mathrm{P}(\mathrm{O}) \mathrm{Ph}_{2}$-moiety. The distance was measured at 3.6012 (11) $\AA$.

The packing of the title compound in the crystal structure is shown in Figure 3.

\section{S2. Experimental}

The compound was obtained commercially (Aldrich). A colourles block suitable for the X-ray diffraction study were taken directly from the provided material.

\section{S3. Refinement}

Carbon-bound H-atoms were placed in calculated positions ( $\mathrm{C}-\mathrm{H} 0.95 \AA)$ and were included in the refinement in the riding model approximation, with $U(\mathrm{H})$ set to $1.2 U_{\mathrm{eq}}(\mathrm{C})$.

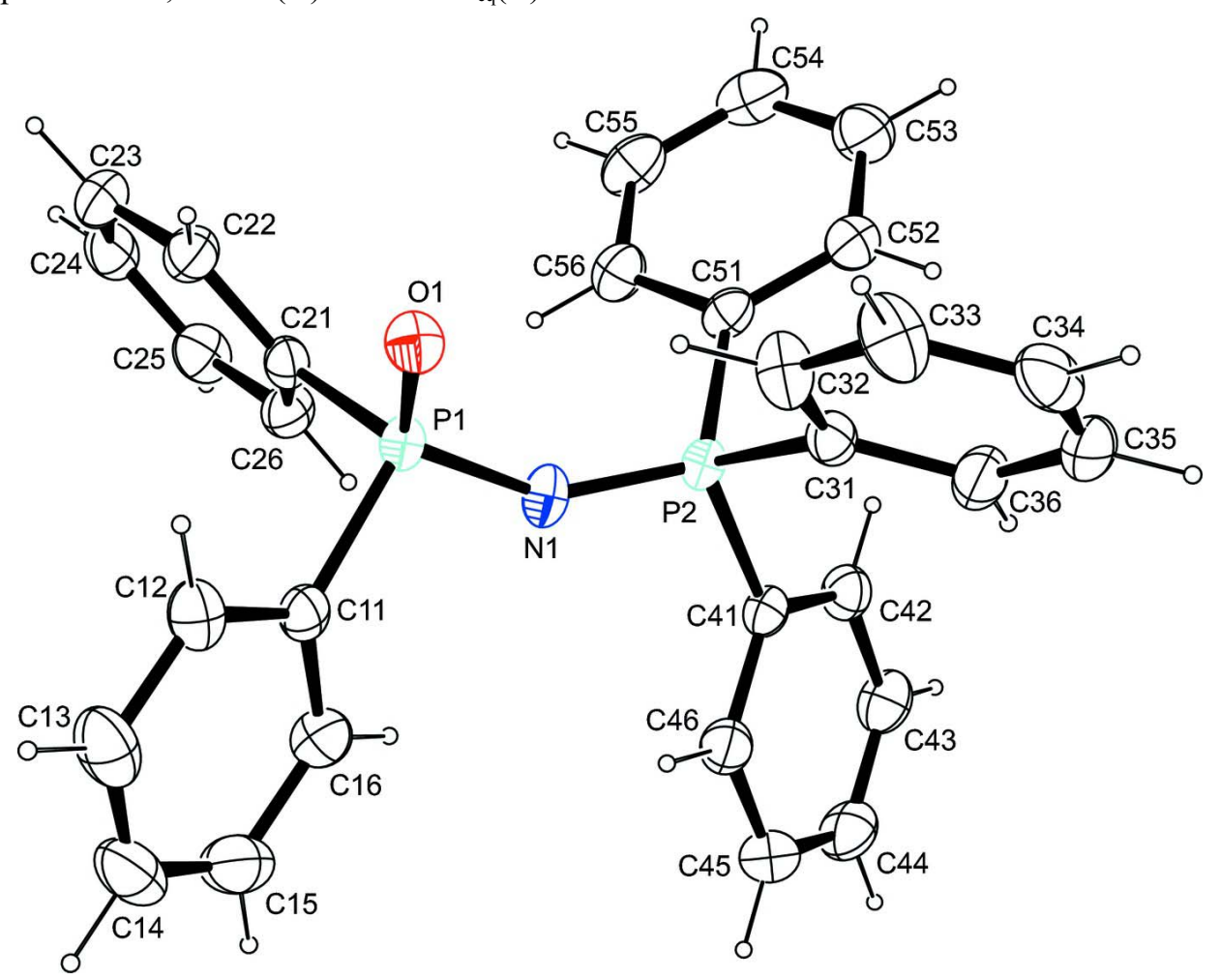

\section{Figure 1}

The molecular structure of the title compound, with anisotropic displacement ellipsoids (drawn at $50 \%$ probability level). 


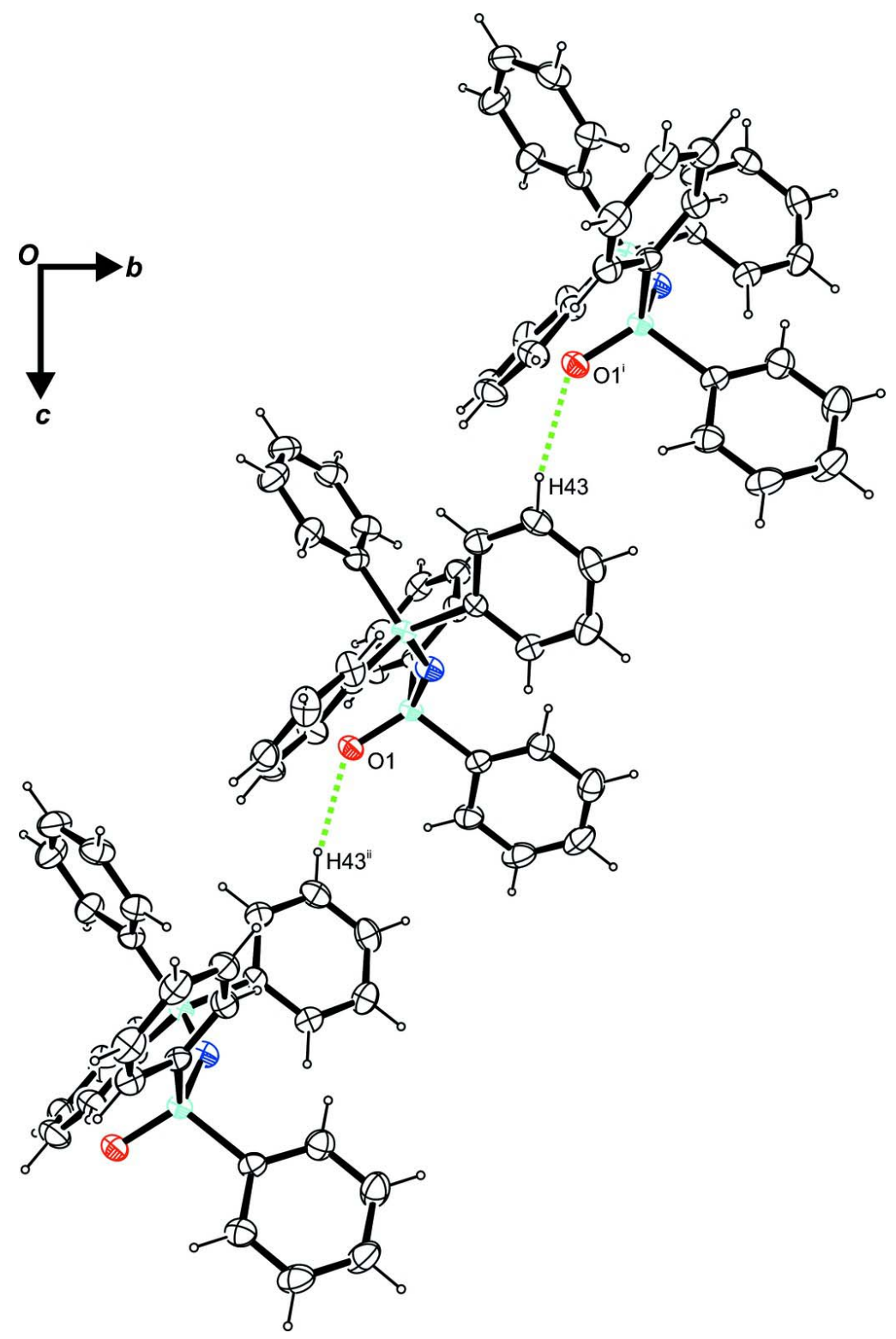

Figure 2

Intermolecular contacts, viewed along [-1 00$]$. Symmetry operators: i $-x+1 / 2, y-1 / 2, z+1 / 2$; ii $-x+1 / 2, y+1 / 2, z-1 / 2$. 


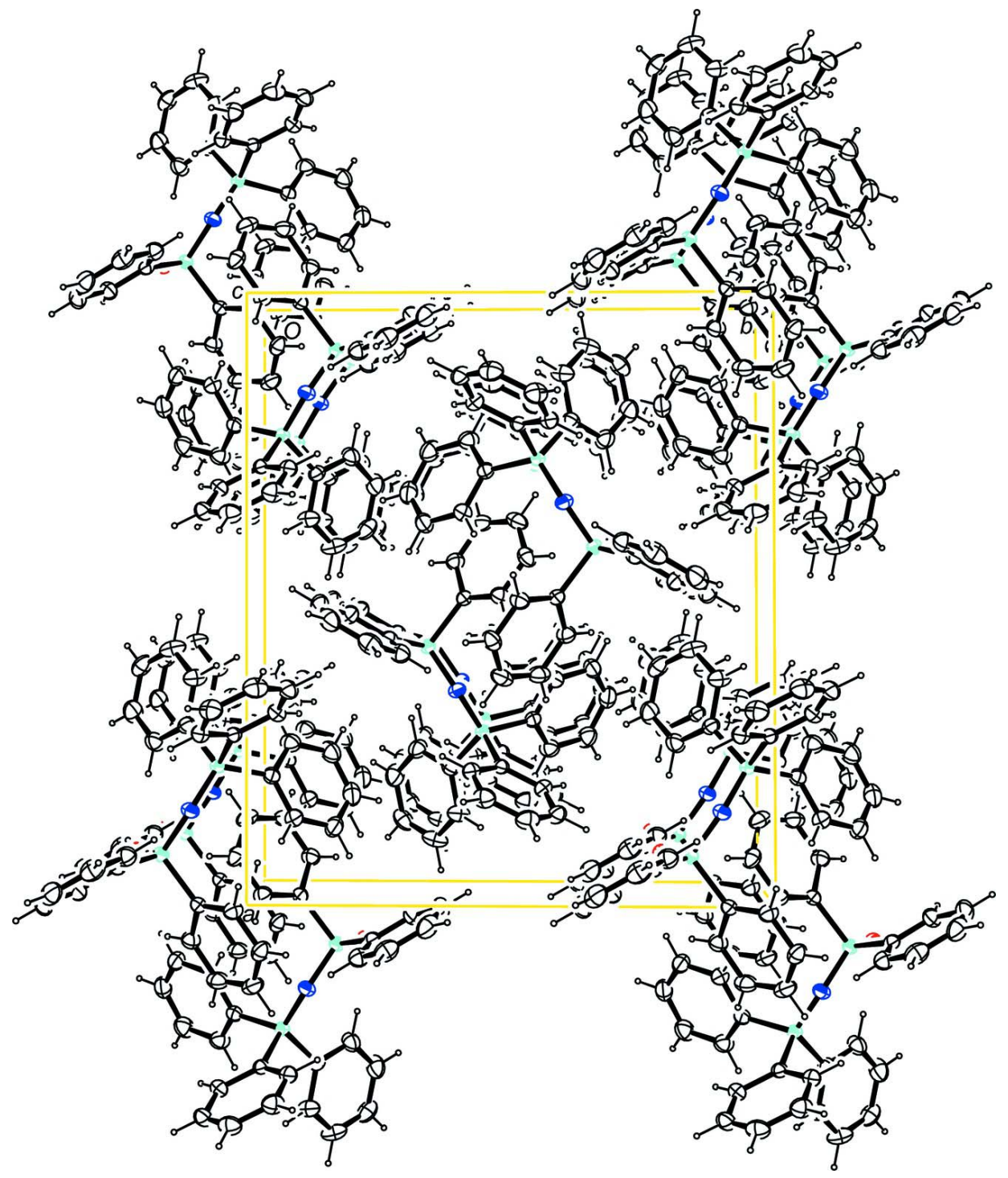

Figure 3

Crystal packing of the title compound, viewed along [ $\left[\begin{array}{lll}0 & 0 & -1\end{array}\right]$ (anisotropic displacement ellipsoids drawn at 50\% probability level).

\section{Diphenylphosphonimidotriphenylphosphorane}

Crystal data

$\mathrm{C}_{30} \mathrm{H}_{25} \mathrm{NOP}_{2}$

$M_{r}=477.45$

Orthorhombic, Pna2 ${ }_{1}$

Hall symbol: P 2c -2n

$a=17.6607$ (12) $\AA$

$b=15.1593(10) \AA$

$c=8.9192(6) \AA$

$V=2387.9(3) \AA^{3}$

$Z=4$

$F(000)=1000$
$D_{\mathrm{x}}=1.328 \mathrm{Mg} \mathrm{m}^{-3}$

Melting point $=442-445 \mathrm{~K}$

Mo $K \alpha$ radiation, $\lambda=0.71073 \AA$

Cell parameters from 9977 reflections

$\theta=2.7-28.3^{\circ}$

$\mu=0.21 \mathrm{~mm}^{-1}$

$T=200 \mathrm{~K}$

Block, colourless

$0.88 \times 0.42 \times 0.31 \mathrm{~mm}$ 


\section{Data collection}

Bruker APEXII CCD

diffractometer

Radiation source: fine-focus sealed tube

Graphite monochromator

$\varphi$ and $\omega$ scans

12511 measured reflections

4498 independent reflections

\section{Refinement}

Refinement on $F^{2}$

Least-squares matrix: full

$R\left[F^{2}>2 \sigma\left(F^{2}\right)\right]=0.027$

$w R\left(F^{2}\right)=0.076$

$S=1.11$

4498 reflections

307 parameters

1 restraint

Primary atom site location: structure-invariant direct methods

Secondary atom site location: difference Fourier map
4348 reflections with $I>2 \sigma(I)$

$R_{\text {int }}=0.021$

$\theta_{\text {max }}=28.3^{\circ}, \theta_{\min }=2.9^{\circ}$

$h=-22 \rightarrow 23$

$k=-20 \rightarrow 16$

$l=-7 \rightarrow 11$

Hydrogen site location: inferred from neighbouring sites

$\mathrm{H}$-atom parameters constrained

$w=1 /\left[\sigma^{2}\left(F_{\mathrm{o}}^{2}\right)+(0.0452 P)^{2}+0.5077 P\right]$

where $P=\left(F_{\mathrm{o}}^{2}+2 F_{\mathrm{c}}^{2}\right) / 3$

$(\Delta / \sigma)_{\max }<0.001$

$\Delta \rho_{\max }=0.21 \mathrm{e} \AA^{-3}$

$\Delta \rho_{\min }=-0.25$ e $\AA^{-3}$

Absolute structure: Flack (1983), 1332 Friedel pairs

Absolute structure parameter: -0.03 (6)

Fractional atomic coordinates and isotropic or equivalent isotropic displacement parameters $\left(\hat{A}^{2}\right)$

\begin{tabular}{lllll}
\hline & $x$ & $y$ & $z$ & $U_{\text {iso }} / U_{\mathrm{eq}}$ \\
\hline P1 & $0.083912(19)$ & $0.16050(2)$ & $0.55082(5)$ & $0.02138(9)$ \\
P2 & $0.227542(18)$ & $0.05280(2)$ & $0.53590(6)$ & $0.02115(9)$ \\
O1 & $0.08330(6)$ & $0.21271(8)$ & $0.40946(15)$ & $0.0276(3)$ \\
N1 & $0.15673(7)$ & $0.10297(9)$ & $0.59418(19)$ & $0.0272(3)$ \\
C11 & $0.06899(9)$ & $0.23246(11)$ & $0.7102(2)$ & $0.0251(3)$ \\
C12 & $0.02931(10)$ & $0.31087(11)$ & $0.6883(3)$ & $0.0327(4)$ \\
H12 & 0.0132 & 0.3268 & 0.5903 & $0.039^{*}$ \\
C13 & $0.01304(11)$ & $0.36602(13)$ & $0.8084(3)$ & $0.0420(5)$ \\
H13 & -0.0139 & 0.4194 & 0.7924 & $0.050^{*}$ \\
C14 & $0.03602(12)$ & $0.34320(14)$ & $0.9502(3)$ & $0.0440(5)$ \\
H14 & 0.0252 & 0.3813 & 1.0321 & $0.053^{*}$ \\
C15 & $0.07484(12)$ & $0.26496(15)$ & $0.9752(3)$ & $0.0426(5)$ \\
H15 & 0.0898 & 0.2489 & 1.0738 & $0.051^{*}$ \\
C16 & $0.09162(10)$ & $0.21044(13)$ & $0.8546(2)$ & $0.0336(4)$ \\
H16 & 0.1190 & 0.1573 & 0.8710 & $0.040^{*}$ \\
C21 & $0.00231(8)$ & $0.08902(9)$ & $0.5576(2)$ & $0.0228(3)$ \\
C22 & $-0.05883(9)$ & $0.10711(11)$ & $0.4654(2)$ & $0.0294(4)$ \\
H22 & -0.0563 & 0.1549 & 0.3968 & $0.035^{*}$ \\
C23 & $-0.12411(10)$ & $0.05546(13)$ & $0.4728(2)$ & $0.0358(4)$ \\
H23 & -0.1663 & 0.0687 & 0.4106 & $0.043^{*}$ \\
C24 & $-0.12731(9)$ & $-0.01467(12)$ & $0.5705(2)$ & $0.0357(4)$ \\
H24 & -0.1714 & -0.0504 & 0.5741 & $0.043^{*}$ \\
C25 & $-0.06683(10)$ & $-0.03348(12)$ & $0.6635(2)$ & $0.0331(4)$ \\
H25 & -0.0693 & -0.0819 & 0.7309 & $0.040^{*}$
\end{tabular}




$\begin{array}{lllll}\text { C26 } & -0.00227(9) & 0.01904(11) & 0.6576(2) & 0.0286(4) \\ \text { H26 } & 0.0391 & 0.0069 & 0.7225 & 0.034^{*} \\ \text { C31 } & 0.29089(9) & 0.11812(11) & 0.4214(2) & 0.0250(3) \\ \text { C32 } & 0.26144(10) & 0.18326(12) & 0.3296(2) & 0.0352(4) \\ \text { H32 } & 0.2087 & 0.1958 & 0.3315 & 0.042^{*} \\ \text { C33 } & 0.30909(12) & 0.23043(13) & 0.2347(3) & 0.0421(5) \\ \text { H33 } & 0.2887 & 0.2747 & 0.1711 & 0.051^{*} \\ \text { C34 } & 0.38560(11) & 0.21312(13) & 0.2327(2) & 0.0378(4) \\ \text { H34 } & 0.4179 & 0.2455 & 0.1676 & 0.045^{*} \\ \text { C35 } & 0.41530(10) & 0.14935(15) & 0.3242(3) & 0.0404(5) \\ \text { H35 } & 0.4681 & 0.1377 & 0.3222 & 0.048^{*} \\ \text { C36 } & 0.36879(10) & 0.10175(14) & 0.4197(2) & 0.0350(4) \\ \text { H36 } & 0.3898 & 0.0581 & 0.4839 & 0.042^{*} \\ \text { C41 } & 0.28149(8) & 0.01741(10) & 0.6966(2) & 0.0222(3) \\ \text { C42 } & 0.31729(9) & -0.06444(11) & 0.7022(2) & 0.0288(4) \\ \text { H42 } & 0.3146 & -0.1031 & 0.6186 & 0.035^{*} \\ \text { C43 } & 0.35690(10) & -0.08954(12) & 0.8295(2) & 0.0340(4) \\ \text { H43 } & 0.3809 & -0.1456 & 0.8334 & 0.041^{*} \\ \text { C44 } & 0.36148(10) & -0.03317(14) & 0.9506(2) & 0.0375(4) \\ \text { H44 } & 0.3886 & -0.0504 & 1.0378 & 0.045^{*} \\ \text { C45 } & 0.32647(11) & 0.04883(13) & 0.9453(2) & 0.0372(4) \\ \text { H45 } & 0.3301 & 0.0878 & 1.0284 & 0.045^{*} \\ \text { C46 } & 0.28624(9) & 0.07380(11) & 0.8190(2) & 0.0290(4) \\ \text { H46 } & 0.2618 & 0.1296 & 0.8161 & 0.035^{*} \\ \text { C51 } & 0.20882(9) & -0.04527(11) & 0.4262(2) & 0.0252(3) \\ \text { C52 } & 0.25999(10) & -0.07830(13) & 0.3230(2) & 0.0335(4) \\ \text { H52 } & 0.3072 & -0.0494 & 0.3088 & 0.040^{*} \\ \text { C53 } & 0.24317(12) & -0.15319(13) & 0.2399(3) & 0.0402(5) \\ \text { H53 } & 0.2786 & -0.1757 & 0.1695 & 0.048^{*} \\ \text { C54 } & 0.17403(12) & -0.19490(12) & 0.2607(3) & 0.0396(5) \\ \text { H54 } & 0.1617 & -0.2456 & 0.2030 & 0.047^{*} \\ \text { C55 } & 0.12324(11) & -0.16321(12) & 0.3645(3) & 0.0369(4) \\ \text { H55 } & 0.0763 & -0.1926 & 0.3792 & 0.044^{*} \\ \text { C56 } & 0.14026(9) & -0.08887(11) & 0.4474(2) & 0.0310(4) \\ \text { H56 } & 0.1050 & -0.0674 & 0.5192 & 0.037^{*} \\ & & & & \end{array}$

Atomic displacement parameters $\left(\AA^{2}\right)$

\begin{tabular}{lllllll}
\hline & $U^{11}$ & $U^{22}$ & $U^{33}$ & $U^{12}$ & $U^{13}$ & $U^{23}$ \\
\hline P1 & $0.01837(15)$ & $0.02043(17)$ & $0.0254(2)$ & $0.00090(11)$ & $0.00122(16)$ & $0.00224(17)$ \\
P2 & $0.01691(14)$ & $0.02080(17)$ & $0.0257(2)$ & $0.00025(12)$ & $-0.00016(17)$ & $0.00197(17)$ \\
O1 & $0.0292(6)$ & $0.0257(6)$ & $0.0280(7)$ & $0.0001(4)$ & $0.0027(5)$ & $0.0053(5)$ \\
N1 & $0.0199(5)$ & $0.0267(6)$ & $0.0351(8)$ & $0.0034(5)$ & $0.0010(5)$ & $0.0037(6)$ \\
C11 & $0.0199(6)$ & $0.0245(8)$ & $0.0308(9)$ & $-0.0035(6)$ & $0.0018(6)$ & $-0.0028(7)$ \\
C12 & $0.0298(8)$ & $0.0289(8)$ & $0.0395(11)$ & $0.0029(6)$ & $0.0048(8)$ & $0.0001(8)$ \\
C13 & $0.0428(10)$ & $0.0290(9)$ & $0.0542(14)$ & $0.0017(8)$ & $0.0125(10)$ & $-0.0085(9)$ \\
C14 & $0.0450(10)$ & $0.0417(11)$ & $0.0452(13)$ & $-0.0110(8)$ & $0.0144(10)$ & $-0.0188(10)$ \\
C15 & $0.0421(10)$ & $0.0524(12)$ & $0.0333(11)$ & $-0.0127(9)$ & $-0.0004(9)$ & $-0.0061(9)$
\end{tabular}




\begin{tabular}{lllllll} 
C16 & $0.0309(8)$ & $0.0361(9)$ & $0.0338(10)$ & $-0.0025(7)$ & $-0.0022(8)$ & $-0.0020(8)$ \\
C21 & $0.0199(6)$ & $0.0213(6)$ & $0.0271(9)$ & $0.0013(5)$ & $0.0022(6)$ & $-0.0025(7)$ \\
C22 & $0.0264(7)$ & $0.0307(8)$ & $0.0312(9)$ & $0.0017(6)$ & $-0.0034(7)$ & $-0.0001(7)$ \\
C23 & $0.0232(7)$ & $0.0444(10)$ & $0.0398(11)$ & $0.0007(7)$ & $-0.0051(7)$ & $-0.0080(9)$ \\
C24 & $0.0265(7)$ & $0.0418(9)$ & $0.0388(11)$ & $-0.0100(6)$ & $0.0079(7)$ & $-0.0124(8)$ \\
C25 & $0.0346(8)$ & $0.0314(8)$ & $0.0332(10)$ & $-0.0077(7)$ & $0.0073(8)$ & $0.0006(8)$ \\
C26 & $0.0258(7)$ & $0.0317(8)$ & $0.0282(9)$ & $-0.0022(6)$ & $0.0002(7)$ & $0.0028(7)$ \\
C31 & $0.0232(7)$ & $0.0257(7)$ & $0.0262(8)$ & $-0.0035(6)$ & $0.0031(6)$ & $-0.0017(7)$ \\
C32 & $0.0318(8)$ & $0.0326(9)$ & $0.0411(12)$ & $0.0050(7)$ & $0.0094(8)$ & $0.0071(8)$ \\
C33 & $0.0481(10)$ & $0.0323(9)$ & $0.0460(12)$ & $0.0030(8)$ & $0.0174(10)$ & $0.0110(9)$ \\
C34 & $0.0417(10)$ & $0.0398(10)$ & $0.0320(10)$ & $-0.0152(8)$ & $0.0124(8)$ & $-0.0041(8)$ \\
C35 & $0.0238(8)$ & $0.0626(13)$ & $0.0348(11)$ & $-0.0106(8)$ & $0.0025(8)$ & $-0.0013(10)$ \\
C36 & $0.0243(7)$ & $0.0480(10)$ & $0.0328(10)$ & $-0.0023(7)$ & $-0.0012(7)$ & $0.0056(8)$ \\
C41 & $0.0186(6)$ & $0.0234(7)$ & $0.0247(8)$ & $-0.0013(5)$ & $-0.0001(6)$ & $0.0030(6)$ \\
C42 & $0.0257(7)$ & $0.0273(8)$ & $0.0335(10)$ & $0.0035(6)$ & $-0.0004(7)$ & $-0.0010(7)$ \\
C43 & $0.0290(8)$ & $0.0324(8)$ & $0.0407(11)$ & $0.0042(6)$ & $-0.0041(8)$ & $0.0091(8)$ \\
C44 & $0.0316(8)$ & $0.0503(11)$ & $0.0305(10)$ & $0.0008(7)$ & $-0.0061(7)$ & $0.0094(9)$ \\
C45 & $0.0346(9)$ & $0.0451(10)$ & $0.0319(10)$ & $-0.0010(7)$ & $-0.0040(8)$ & $-0.0076(9)$ \\
C46 & $0.0255(7)$ & $0.0279(8)$ & $0.0335(10)$ & $0.0004(6)$ & $-0.0005(7)$ & $-0.0030(8)$ \\
C51 & $0.0232(7)$ & $0.0239(7)$ & $0.0284(9)$ & $0.0016(5)$ & $-0.0052(6)$ & $0.0014(6)$ \\
C52 & $0.0289(8)$ & $0.0379(9)$ & $0.0339(11)$ & $0.0013(7)$ & $-0.0019(7)$ & $-0.0064(8)$ \\
C53 & $0.0417(10)$ & $0.0382(10)$ & $0.0407(12)$ & $0.0087(8)$ & $-0.0027(9)$ & $-0.0109(9)$ \\
C54 & $0.0471(10)$ & $0.0272(8)$ & $0.0443(12)$ & $0.0033(7)$ & $-0.0163(9)$ & $-0.0032(8)$ \\
C55 & $0.0346(8)$ & $0.0282(8)$ & $0.0480(12)$ & $-0.0054(7)$ & $-0.0104(9)$ & $0.0039(8)$ \\
C56 & $0.0265(7)$ & $0.0286(8)$ & $0.0379(10)$ & $-0.0019(6)$ & $-0.0023(7)$ & $0.0012(8)$ \\
& & & & & & \\
\hline
\end{tabular}

Geometric parameters $\left(\AA,{ }^{\circ}\right)$

\begin{tabular}{llll}
\hline $\mathrm{P} 1-\mathrm{O} 1$ & $1.4887(13)$ & $\mathrm{C} 32-\mathrm{C} 33$ & $1.392(3)$ \\
$\mathrm{P} 1-\mathrm{N} 1$ & $1.6014(13)$ & $\mathrm{C} 32-\mathrm{H} 32$ & 0.9500 \\
$\mathrm{P} 1-\mathrm{C} 21$ & $1.8040(15)$ & $\mathrm{C} 33-\mathrm{C} 34$ & $1.377(3)$ \\
$\mathrm{P} 1-\mathrm{C} 11$ & $1.8109(18)$ & $\mathrm{C} 33-\mathrm{H} 33$ & 0.9500 \\
$\mathrm{P} 2-\mathrm{N} 1$ & $1.5532(13)$ & $\mathrm{C} 34-\mathrm{C} 35$ & $1.370(3)$ \\
$\mathrm{P} 2-\mathrm{C} 41$ & $1.8027(17)$ & $\mathrm{C} 34-\mathrm{H} 34$ & 0.9500 \\
$\mathrm{P} 2-\mathrm{C} 31$ & $1.8099(17)$ & $\mathrm{C} 35-\mathrm{C} 36$ & $1.386(3)$ \\
$\mathrm{P} 2-\mathrm{C} 51$ & $1.8099(17)$ & $\mathrm{C} 35-\mathrm{H} 35$ & 0.9500 \\
$\mathrm{C} 11-\mathrm{C} 16$ & $1.389(3)$ & $\mathrm{C} 36-\mathrm{H} 36$ & 0.9500 \\
$\mathrm{C} 11-\mathrm{C} 12$ & $1.394(2)$ & $\mathrm{C} 41-\mathrm{C} 46$ & $1.389(2)$ \\
$\mathrm{C} 12-\mathrm{C} 13$ & $1.389(3)$ & $\mathrm{C} 41-\mathrm{C} 42$ & $1.394(2)$ \\
$\mathrm{C} 12-\mathrm{H} 12$ & 0.9500 & $\mathrm{C} 42-\mathrm{C} 43$ & $1.387(3)$ \\
$\mathrm{C} 13-\mathrm{C} 14$ & $1.373(4)$ & $\mathrm{C} 42-\mathrm{H} 42$ & 0.9500 \\
$\mathrm{C} 13-\mathrm{H} 13$ & 0.9500 & $\mathrm{C} 43-\mathrm{C} 44$ & $1.380(3)$ \\
$\mathrm{C} 14-\mathrm{C} 15$ & $1.388(3)$ & $\mathrm{C} 43-\mathrm{H} 43$ & 0.9500 \\
$\mathrm{C} 14-\mathrm{H} 14$ & 0.9500 & $\mathrm{C} 44-\mathrm{C} 45$ & $1.389(3)$ \\
$\mathrm{C} 15-\mathrm{C} 16$ & $1.388(3)$ & $\mathrm{C} 44-\mathrm{H} 44$ & 0.9500 \\
$\mathrm{C} 15-\mathrm{H} 15$ & 0.9500 & $\mathrm{C} 45-\mathrm{C} 46$ & $1.384(3)$ \\
$\mathrm{C} 16-\mathrm{H} 16$ & 0.9500 & $\mathrm{C} 45-\mathrm{H} 45$ & 0.9500 \\
$\mathrm{C} 21-\mathrm{C} 22$ & $1.385(2)$ & $\mathrm{C} 46-\mathrm{H} 46$ & 0.9500
\end{tabular}




\begin{tabular}{|c|c|c|c|}
\hline $\mathrm{C} 21-\mathrm{C} 26$ & $1.388(2)$ & $\mathrm{C} 51-\mathrm{C} 52$ & $1.384(3)$ \\
\hline $\mathrm{C} 22-\mathrm{C} 23$ & $1.395(2)$ & $\mathrm{C} 51-\mathrm{C} 56$ & $1.392(2)$ \\
\hline $\mathrm{C} 22-\mathrm{H} 22$ & 0.9500 & $\mathrm{C} 52-\mathrm{C} 53$ & $1.388(3)$ \\
\hline $\mathrm{C} 23-\mathrm{C} 24$ & $1.376(3)$ & C52-H52 & 0.9500 \\
\hline $\mathrm{C} 23-\mathrm{H} 23$ & 0.9500 & $\mathrm{C} 53-\mathrm{C} 54$ & $1.387(3)$ \\
\hline $\mathrm{C} 24-\mathrm{C} 25$ & $1.382(3)$ & C53-H53 & 0.9500 \\
\hline $\mathrm{C} 24-\mathrm{H} 24$ & 0.9500 & C54-C55 & $1.375(3)$ \\
\hline $\mathrm{C} 25-\mathrm{C} 26$ & $1.392(2)$ & C54-H54 & 0.9500 \\
\hline $\mathrm{C} 25-\mathrm{H} 25$ & 0.9500 & $\mathrm{C} 55-\mathrm{C} 56$ & $1.381(3)$ \\
\hline $\mathrm{C} 26-\mathrm{H} 26$ & 0.9500 & $\mathrm{C} 55-\mathrm{H} 55$ & 0.9500 \\
\hline $\mathrm{C} 31-\mathrm{C} 32$ & $1.384(3)$ & $\mathrm{C} 56-\mathrm{H} 56$ & 0.9500 \\
\hline $\mathrm{C} 31-\mathrm{C} 36$ & $1.398(2)$ & & \\
\hline $\mathrm{O} 1-\mathrm{P} 1-\mathrm{N} 1$ & $120.00(8)$ & $\mathrm{C} 31-\mathrm{C} 32-\mathrm{C} 33$ & $119.94(17)$ \\
\hline $\mathrm{O} 1-\mathrm{P} 1-\mathrm{C} 21$ & $110.00(8)$ & $\mathrm{C} 31-\mathrm{C} 32-\mathrm{H} 32$ & 120.0 \\
\hline $\mathrm{N} 1-\mathrm{P} 1-\mathrm{C} 21$ & $107.83(7)$ & $\mathrm{C} 33-\mathrm{C} 32-\mathrm{H} 32$ & 120.0 \\
\hline $\mathrm{O} 1-\mathrm{P} 1-\mathrm{C} 11$ & $110.08(7)$ & $\mathrm{C} 34-\mathrm{C} 33-\mathrm{C} 32$ & $120.2(2)$ \\
\hline $\mathrm{N} 1-\mathrm{P} 1-\mathrm{C} 11$ & $104.80(8)$ & $\mathrm{C} 34-\mathrm{C} 33-\mathrm{H} 33$ & 119.9 \\
\hline $\mathrm{C} 21-\mathrm{P} 1-\mathrm{C} 11$ & $102.66(7)$ & $\mathrm{C} 32-\mathrm{C} 33-\mathrm{H} 33$ & 119.9 \\
\hline $\mathrm{N} 1-\mathrm{P} 2-\mathrm{C} 41$ & $107.77(8)$ & $\mathrm{C} 35-\mathrm{C} 34-\mathrm{C} 33$ & $120.16(18)$ \\
\hline $\mathrm{N} 1-\mathrm{P} 2-\mathrm{C} 31$ & $114.75(7)$ & $\mathrm{C} 35-\mathrm{C} 34-\mathrm{H} 34$ & 119.9 \\
\hline $\mathrm{C} 41-\mathrm{P} 2-\mathrm{C} 31$ & $106.55(7)$ & $\mathrm{C} 33-\mathrm{C} 34-\mathrm{H} 34$ & 119.9 \\
\hline $\mathrm{N} 1-\mathrm{P} 2-\mathrm{C} 51$ & $115.84(7)$ & $\mathrm{C} 34-\mathrm{C} 35-\mathrm{C} 36$ & $120.47(17)$ \\
\hline $\mathrm{C} 41-\mathrm{P} 2-\mathrm{C} 51$ & $106.37(8)$ & $\mathrm{C} 34-\mathrm{C} 35-\mathrm{H} 35$ & 119.8 \\
\hline $\mathrm{C} 31-\mathrm{P} 2-\mathrm{C} 51$ & $104.91(8)$ & $\mathrm{C} 36-\mathrm{C} 35-\mathrm{H} 35$ & 119.8 \\
\hline $\mathrm{P} 2-\mathrm{N} 1-\mathrm{P} 1$ & $146.35(12)$ & $\mathrm{C} 35-\mathrm{C} 36-\mathrm{C} 31$ & $119.81(18)$ \\
\hline $\mathrm{C} 16-\mathrm{C} 11-\mathrm{C} 12$ & $118.64(17)$ & $\mathrm{C} 35-\mathrm{C} 36-\mathrm{H} 36$ & 120.1 \\
\hline $\mathrm{C} 16-\mathrm{C} 11-\mathrm{P} 1$ & $122.75(13)$ & $\mathrm{C} 31-\mathrm{C} 36-\mathrm{H} 36$ & 120.1 \\
\hline $\mathrm{C} 12-\mathrm{C} 11-\mathrm{P} 1$ & $118.51(15)$ & $\mathrm{C} 46-\mathrm{C} 41-\mathrm{C} 42$ & $119.50(16)$ \\
\hline $\mathrm{C} 13-\mathrm{C} 12-\mathrm{C} 11$ & $120.6(2)$ & $\mathrm{C} 46-\mathrm{C} 41-\mathrm{P} 2$ & $118.28(12)$ \\
\hline $\mathrm{C} 13-\mathrm{C} 12-\mathrm{H} 12$ & 119.7 & $\mathrm{C} 42-\mathrm{C} 41-\mathrm{P} 2$ & $122.22(14)$ \\
\hline $\mathrm{C} 11-\mathrm{C} 12-\mathrm{H} 12$ & 119.7 & $\mathrm{C} 43-\mathrm{C} 42-\mathrm{C} 41$ & $120.15(18)$ \\
\hline $\mathrm{C} 14-\mathrm{C} 13-\mathrm{C} 12$ & $119.86(19)$ & $\mathrm{C} 43-\mathrm{C} 42-\mathrm{H} 42$ & 119.9 \\
\hline $\mathrm{C} 14-\mathrm{C} 13-\mathrm{H} 13$ & 120.1 & $\mathrm{C} 41-\mathrm{C} 42-\mathrm{H} 42$ & 119.9 \\
\hline $\mathrm{C} 12-\mathrm{C} 13-\mathrm{H} 13$ & 120.1 & $\mathrm{C} 44-\mathrm{C} 43-\mathrm{C} 42$ & $120.04(17)$ \\
\hline $\mathrm{C} 13-\mathrm{C} 14-\mathrm{C} 15$ & $120.6(2)$ & $\mathrm{C} 44-\mathrm{C} 43-\mathrm{H} 43$ & 120.0 \\
\hline $\mathrm{C} 13-\mathrm{C} 14-\mathrm{H} 14$ & 119.7 & $\mathrm{C} 42-\mathrm{C} 43-\mathrm{H} 43$ & 120.0 \\
\hline $\mathrm{C} 15-\mathrm{C} 14-\mathrm{H} 14$ & 119.7 & $\mathrm{C} 43-\mathrm{C} 44-\mathrm{C} 45$ & $120.09(18)$ \\
\hline $\mathrm{C} 14-\mathrm{C} 15-\mathrm{C} 16$ & $119.3(2)$ & $\mathrm{C} 43-\mathrm{C} 44-\mathrm{H} 44$ & 120.0 \\
\hline $\mathrm{C} 14-\mathrm{C} 15-\mathrm{H} 15$ & 120.3 & $\mathrm{C} 45-\mathrm{C} 44-\mathrm{H} 44$ & 120.0 \\
\hline $\mathrm{C} 16-\mathrm{C} 15-\mathrm{H} 15$ & 120.3 & $\mathrm{C} 46-\mathrm{C} 45-\mathrm{C} 44$ & $120.07(18)$ \\
\hline $\mathrm{C} 15-\mathrm{C} 16-\mathrm{C} 11$ & $120.92(18)$ & $\mathrm{C} 46-\mathrm{C} 45-\mathrm{H} 45$ & 120.0 \\
\hline $\mathrm{C} 15-\mathrm{C} 16-\mathrm{H} 16$ & 119.5 & $\mathrm{C} 44-\mathrm{C} 45-\mathrm{H} 45$ & 120.0 \\
\hline $\mathrm{C} 11-\mathrm{C} 16-\mathrm{H} 16$ & 119.5 & $\mathrm{C} 45-\mathrm{C} 46-\mathrm{C} 41$ & $120.15(16)$ \\
\hline $\mathrm{C} 22-\mathrm{C} 21-\mathrm{C} 26$ & $119.16(14)$ & $\mathrm{C} 45-\mathrm{C} 46-\mathrm{H} 46$ & 119.9 \\
\hline $\mathrm{C} 22-\mathrm{C} 21-\mathrm{P} 1$ & $118.95(13)$ & $\mathrm{C} 41-\mathrm{C} 46-\mathrm{H} 46$ & 119.9 \\
\hline $\mathrm{C} 26-\mathrm{C} 21-\mathrm{P} 1$ & $121.82(12)$ & $\mathrm{C} 52-\mathrm{C} 51-\mathrm{C} 56$ & 119.09 (16) \\
\hline $\mathrm{C} 21-\mathrm{C} 22-\mathrm{C} 23$ & $120.35(17)$ & $\mathrm{C} 52-\mathrm{C} 51-\mathrm{P} 2$ & $122.51(13)$ \\
\hline
\end{tabular}




\begin{tabular}{|c|c|c|c|}
\hline $\mathrm{C} 21-\mathrm{C} 22-\mathrm{H} 22$ & 119.8 & $\mathrm{C} 56-\mathrm{C} 51-\mathrm{P} 2$ & $118.39(14)$ \\
\hline $\mathrm{C} 23-\mathrm{C} 22-\mathrm{H} 22$ & 119.8 & $\mathrm{C} 51-\mathrm{C} 52-\mathrm{C} 53$ & $120.76(17)$ \\
\hline $\mathrm{C} 24-\mathrm{C} 23-\mathrm{C} 22$ & $119.84(17)$ & $\mathrm{C} 51-\mathrm{C} 52-\mathrm{H} 52$ & 119.6 \\
\hline $\mathrm{C} 24-\mathrm{C} 23-\mathrm{H} 23$ & 120.1 & $\mathrm{C} 53-\mathrm{C} 52-\mathrm{H} 52$ & 119.6 \\
\hline $\mathrm{C} 22-\mathrm{C} 23-\mathrm{H} 23$ & 120.1 & $\mathrm{C} 54-\mathrm{C} 53-\mathrm{C} 52$ & $119.33(19)$ \\
\hline $\mathrm{C} 23-\mathrm{C} 24-\mathrm{C} 25$ & $120.51(15)$ & $\mathrm{C} 54-\mathrm{C} 53-\mathrm{H} 53$ & 120.3 \\
\hline $\mathrm{C} 23-\mathrm{C} 24-\mathrm{H} 24$ & 119.7 & $\mathrm{C} 52-\mathrm{C} 53-\mathrm{H} 53$ & 120.3 \\
\hline $\mathrm{C} 25-\mathrm{C} 24-\mathrm{H} 24$ & 119.7 & $\mathrm{C} 55-\mathrm{C} 54-\mathrm{C} 53$ & $120.31(18)$ \\
\hline $\mathrm{C} 24-\mathrm{C} 25-\mathrm{C} 26$ & $119.50(18)$ & $\mathrm{C} 55-\mathrm{C} 54-\mathrm{H} 54$ & 119.8 \\
\hline $\mathrm{C} 24-\mathrm{C} 25-\mathrm{H} 25$ & 120.3 & $\mathrm{C} 53-\mathrm{C} 54-\mathrm{H} 54$ & 119.8 \\
\hline $\mathrm{C} 26-\mathrm{C} 25-\mathrm{H} 25$ & 120.3 & $\mathrm{C} 54-\mathrm{C} 55-\mathrm{C} 56$ & $120.23(18)$ \\
\hline $\mathrm{C} 21-\mathrm{C} 26-\mathrm{C} 25$ & $120.63(16)$ & $\mathrm{C} 54-\mathrm{C} 55-\mathrm{H} 55$ & 119.9 \\
\hline $\mathrm{C} 21-\mathrm{C} 26-\mathrm{H} 26$ & 119.7 & $\mathrm{C} 56-\mathrm{C} 55-\mathrm{H} 55$ & 119.9 \\
\hline $\mathrm{C} 25-\mathrm{C} 26-\mathrm{H} 26$ & 119.7 & $\mathrm{C} 55-\mathrm{C} 56-\mathrm{C} 51$ & $120.27(18)$ \\
\hline $\mathrm{C} 32-\mathrm{C} 31-\mathrm{C} 36$ & $119.36(16)$ & $\mathrm{C} 55-\mathrm{C} 56-\mathrm{H} 56$ & 119.9 \\
\hline $\mathrm{C} 32-\mathrm{C} 31-\mathrm{P} 2$ & $119.46(12)$ & $\mathrm{C} 51-\mathrm{C} 56-\mathrm{H} 56$ & 119.9 \\
\hline $\mathrm{C} 36-\mathrm{C} 31-\mathrm{P} 2$ & $121.14(14)$ & & \\
\hline $\mathrm{C} 41-\mathrm{P} 2-\mathrm{N} 1-\mathrm{P} 1$ & $173.79(15)$ & $\mathrm{C} 41-\mathrm{P} 2-\mathrm{C} 31-\mathrm{C} 36$ & $29.82(17)$ \\
\hline $\mathrm{C} 31-\mathrm{P} 2-\mathrm{N} 1-\mathrm{P} 1$ & $55.30(19)$ & $\mathrm{C} 51-\mathrm{P} 2-\mathrm{C} 31-\mathrm{C} 36$ & $-82.72(17)$ \\
\hline $\mathrm{C} 51-\mathrm{P} 2-\mathrm{N} 1-\mathrm{P} 1$ & $-67.26(19)$ & $\mathrm{C} 36-\mathrm{C} 31-\mathrm{C} 32-\mathrm{C} 33$ & $1.3(3)$ \\
\hline $\mathrm{O} 1-\mathrm{P} 1-\mathrm{N} 1-\mathrm{P} 2$ & $-25.4(2)$ & $\mathrm{P} 2-\mathrm{C} 31-\mathrm{C} 32-\mathrm{C} 33$ & $-176.30(16)$ \\
\hline $\mathrm{C} 21-\mathrm{P} 1-\mathrm{N} 1-\mathrm{P} 2$ & $101.52(17)$ & $\mathrm{C} 31-\mathrm{C} 32-\mathrm{C} 33-\mathrm{C} 34$ & $-0.6(3)$ \\
\hline $\mathrm{C} 11-\mathrm{P} 1-\mathrm{N} 1-\mathrm{P} 2$ & $-149.62(16)$ & $\mathrm{C} 32-\mathrm{C} 33-\mathrm{C} 34-\mathrm{C} 35$ & $0.0(3)$ \\
\hline $\mathrm{O} 1-\mathrm{P} 1-\mathrm{C} 11-\mathrm{C} 16$ & $-157.41(13)$ & $\mathrm{C} 33-\mathrm{C} 34-\mathrm{C} 35-\mathrm{C} 36$ & $-0.1(3)$ \\
\hline $\mathrm{N} 1-\mathrm{P} 1-\mathrm{C} 11-\mathrm{C} 16$ & $-27.08(16)$ & $\mathrm{C} 34-\mathrm{C} 35-\mathrm{C} 36-\mathrm{C} 31$ & $0.8(3)$ \\
\hline $\mathrm{C} 21-\mathrm{P} 1-\mathrm{C} 11-\mathrm{C} 16$ & $85.50(15)$ & $\mathrm{C} 32-\mathrm{C} 31-\mathrm{C} 36-\mathrm{C} 35$ & $-1.4(3)$ \\
\hline $\mathrm{O} 1-\mathrm{P} 1-\mathrm{C} 11-\mathrm{C} 12$ & $26.37(15)$ & $\mathrm{P} 2-\mathrm{C} 31-\mathrm{C} 36-\mathrm{C} 35$ & $176.14(16)$ \\
\hline $\mathrm{N} 1-\mathrm{P} 1-\mathrm{C} 11-\mathrm{C} 12$ & $156.70(13)$ & $\mathrm{N} 1-\mathrm{P} 2-\mathrm{C} 41-\mathrm{C} 46$ & $-38.07(14)$ \\
\hline $\mathrm{C} 21-\mathrm{P} 1-\mathrm{C} 11-\mathrm{C} 12$ & $-90.72(14)$ & $\mathrm{C} 31-\mathrm{P} 2-\mathrm{C} 41-\mathrm{C} 46$ & $85.57(13)$ \\
\hline $\mathrm{C} 16-\mathrm{C} 11-\mathrm{C} 12-\mathrm{C} 13$ & $0.3(2)$ & $\mathrm{C} 51-\mathrm{P} 2-\mathrm{C} 41-\mathrm{C} 46$ & $-162.90(13)$ \\
\hline $\mathrm{P} 1-\mathrm{C} 11-\mathrm{C} 12-\mathrm{C} 13$ & $176.66(14)$ & $\mathrm{N} 1-\mathrm{P} 2-\mathrm{C} 41-\mathrm{C} 42$ & $141.06(13)$ \\
\hline $\mathrm{C} 11-\mathrm{C} 12-\mathrm{C} 13-\mathrm{C} 14$ & $-0.2(3)$ & $\mathrm{C} 31-\mathrm{P} 2-\mathrm{C} 41-\mathrm{C} 42$ & $-95.30(14)$ \\
\hline $\mathrm{C} 12-\mathrm{C} 13-\mathrm{C} 14-\mathrm{C} 15$ & $-0.6(3)$ & $\mathrm{C} 51-\mathrm{P} 2-\mathrm{C} 41-\mathrm{C} 42$ & $16.23(16)$ \\
\hline $\mathrm{C} 13-\mathrm{C} 14-\mathrm{C} 15-\mathrm{C} 16$ & $1.2(3)$ & $\mathrm{C} 46-\mathrm{C} 41-\mathrm{C} 42-\mathrm{C} 43$ & $0.5(2)$ \\
\hline $\mathrm{C} 14-\mathrm{C} 15-\mathrm{C} 16-\mathrm{C} 11$ & $-1.1(3)$ & $\mathrm{P} 2-\mathrm{C} 41-\mathrm{C} 42-\mathrm{C} 43$ & $-178.67(13)$ \\
\hline $\mathrm{C} 12-\mathrm{C} 11-\mathrm{C} 16-\mathrm{C} 15$ & $0.3(3)$ & $\mathrm{C} 41-\mathrm{C} 42-\mathrm{C} 43-\mathrm{C} 44$ & $-0.5(3)$ \\
\hline $\mathrm{P} 1-\mathrm{C} 11-\mathrm{C} 16-\mathrm{C} 15$ & $-175.88(14)$ & $\mathrm{C} 42-\mathrm{C} 43-\mathrm{C} 44-\mathrm{C} 45$ & $0.0(3)$ \\
\hline $\mathrm{O} 1-\mathrm{P} 1-\mathrm{C} 21-\mathrm{C} 22$ & $-18.58(16)$ & $\mathrm{C} 43-\mathrm{C} 44-\mathrm{C} 45-\mathrm{C} 46$ & $0.7(3)$ \\
\hline $\mathrm{N} 1-\mathrm{P} 1-\mathrm{C} 21-\mathrm{C} 22$ & $-151.12(14)$ & $\mathrm{C} 44-\mathrm{C} 45-\mathrm{C} 46-\mathrm{C} 41$ & $-0.8(3)$ \\
\hline $\mathrm{C} 11-\mathrm{P} 1-\mathrm{C} 21-\mathrm{C} 22$ & $98.56(15)$ & $\mathrm{C} 42-\mathrm{C} 41-\mathrm{C} 46-\mathrm{C} 45$ & $0.2(2)$ \\
\hline $\mathrm{O} 1-\mathrm{P} 1-\mathrm{C} 21-\mathrm{C} 26$ & $164.52(14)$ & $\mathrm{P} 2-\mathrm{C} 41-\mathrm{C} 46-\mathrm{C} 45$ & $179.36(14)$ \\
\hline $\mathrm{N} 1-\mathrm{P} 1-\mathrm{C} 21-\mathrm{C} 26$ & $31.99(17)$ & $\mathrm{N} 1-\mathrm{P} 2-\mathrm{C} 51-\mathrm{C} 52$ & $155.63(15)$ \\
\hline $\mathrm{C} 11-\mathrm{P} 1-\mathrm{C} 21-\mathrm{C} 26$ & $-78.33(15)$ & $\mathrm{C} 41-\mathrm{P} 2-\mathrm{C} 51-\mathrm{C} 52$ & $-84.66(17)$ \\
\hline $\mathrm{C} 26-\mathrm{C} 21-\mathrm{C} 22-\mathrm{C} 23$ & $-0.1(3)$ & $\mathrm{C} 31-\mathrm{P} 2-\mathrm{C} 51-\mathrm{C} 52$ & $28.00(17)$ \\
\hline $\mathrm{P} 1-\mathrm{C} 21-\mathrm{C} 22-\mathrm{C} 23$ & $-177.12(14)$ & $\mathrm{N} 1-\mathrm{P} 2-\mathrm{C} 51-\mathrm{C} 56$ & $-24.50(18)$ \\
\hline $\mathrm{C} 21-\mathrm{C} 22-\mathrm{C} 23-\mathrm{C} 24$ & $-1.0(3)$ & $\mathrm{C} 41-\mathrm{P} 2-\mathrm{C} 51-\mathrm{C} 56$ & $95.21(15)$ \\
\hline $\mathrm{C} 22-\mathrm{C} 23-\mathrm{C} 24-\mathrm{C} 25$ & $1.2(3)$ & $\mathrm{C} 31-\mathrm{P} 2-\mathrm{C} 51-\mathrm{C} 56$ & $-152.12(14)$ \\
\hline
\end{tabular}




\section{supporting information}

$$
\begin{aligned}
& \mathrm{C} 23-\mathrm{C} 24-\mathrm{C} 25-\mathrm{C} 26 \\
& \mathrm{C} 22-\mathrm{C} 21-\mathrm{C} 26-\mathrm{C} 25 \\
& \mathrm{P} 1-\mathrm{C} 21-\mathrm{C} 26-\mathrm{C} 25 \\
& \mathrm{C} 24-\mathrm{C} 25-\mathrm{C} 26-\mathrm{C} 21 \\
& \mathrm{~N} 1-\mathrm{P} 2-\mathrm{C} 31-\mathrm{C} 32 \\
& \mathrm{C} 41-\mathrm{P} 2-\mathrm{C} 31-\mathrm{C} 32 \\
& \mathrm{C} 51-\mathrm{P} 2-\mathrm{C} 31-\mathrm{C} 32
\end{aligned}
$$

$-0.1(3)$

$1.2(3)$

$178.09(14)$

$-1.1(3)$

$-33.42(18)$

$-152.60(15)$

$94.86(16)$

$148.99(15)$

$$
\begin{aligned}
& \mathrm{C} 56-\mathrm{C} 51-\mathrm{C} 52-\mathrm{C} 53 \\
& \text { P2-C51-C52-C53 } \\
& \text { C51-C } 52-\mathrm{C} 53-\mathrm{C} 54 \\
& \text { C52-C53-C54-C55 } \\
& \text { C53-C } 54-\mathrm{C} 55-\mathrm{C} 56 \\
& \text { C54-C } 55-\mathrm{C} 56-\mathrm{C} 51 \\
& \text { C52-C } 51-\mathrm{C} 56-\mathrm{C} 55 \\
& \text { P2-C51-C } 56-\mathrm{C} 55
\end{aligned}
$$

$0.8(3)$

$-179.31(16)$

$0.3(3)$

$-1.1(3)$

$0.9(3)$

$0.2(3)$

$-1.0(3)$

$179.07(15)$

Hydrogen-bond geometry $\left(A,{ }^{\circ}\right)$

\begin{tabular}{lllll}
\hline$D-\mathrm{H} \cdots A$ & $D-\mathrm{H}$ & $\mathrm{H} \cdots A$ & $D \cdots A$ & $D-\mathrm{H} \cdots A$ \\
\hline $\mathrm{C} 32-\mathrm{H} 32 \cdots \mathrm{O} 1$ & 0.95 & 2.34 & $3.257(2)$ & 163 \\
$\mathrm{C} 43-\mathrm{H} 43 \cdots \mathrm{O} 1^{\mathrm{i}}$ & 0.95 & 2.34 & $3.257(2)$ & 162 \\
$\mathrm{C} 45-\mathrm{H} 45 \cdots C g 1^{\mathrm{ii}}$ & 0.95 & 2.92 & $3.846(2)$ & 165 \\
$\mathrm{C} 55-\mathrm{H} 55 \cdots C g 2^{\mathrm{ii}}$ & 0.95 & 2.73 & $3.644(2)$ & 163 \\
\hline
\end{tabular}

Symmetry codes: (i) $-x+1 / 2, y-1 / 2, z+1 / 2$; (ii) $x, y, z+1$; (iii) $-x,-y, z-1 / 2$. 